\title{
Leptophilic-portal dark matter in the light of AMS-02 positron excess
}

\author{
Sayan Ghosh $\odot,{ }^{1, *}$ Amit Dutta Banik $\odot,{ }^{2, \dagger}$ Eung Jin Chun, ${ }^{3, *}$ and Debasish Majumdar ${ }^{1, \S}$ \\ ${ }^{1}$ Saha Insitute of Nuclear Physics, HBNI, I/AF Bidhannagar, Kolkata-700 064, India \\ ${ }^{2}$ Key Laboratory of Quark and Lepton Physics (MoE) and Institute of Particle Physics, \\ Central China Normal University, Wuhan 430079, China \\ ${ }^{3}$ Korea Institute for Advanced Study, 85 Hoegiro, Seoul 02455, Republic of Korea
}

(Received 24 June 2021; accepted 9 September 2021; published 13 October 2021)

\begin{abstract}
We revisit dark matter annihilation as an explanation of the positron excess reported recently by the AMS-02 satellite-borne experiment. To this end, we propose a particle dark matter model by considering a two Higgs doublet model (2HDM) extended with an additional singlet boson and a singlet fermion. The additional (light) boson mixes with the pseudoscalar inherent in the 2HDM, and the singlet fermion, which is the dark matter candidate, annihilates via this bosonic portal. The dark matter candidate is made leptophilic by choosing the lepton-specific $2 \mathrm{HDM}$ and a suitable high value of $\tan \beta$. We identify the model parameter space which explains the muon g-2 anomaly while evading the experimental constraints. After establishing the viability of the singlet fermion to be a dark matter candidate, we calculate the positron excess produced from its annihilation to the light bosons which primarily decay to muons. Incorporating the Sommerfeld effect caused by the light mediator and an appropriate boost factor, we find that our proposed model can satisfactorily explain the positron fraction excess as well as the positron spectrum data reported by the AMS-02 experiment.
\end{abstract}

DOI: 10.1103/PhysRevD.104.075016

\section{INTRODUCTION}

The existence of dark matter in the Universe has now been established principally through their gravitational effects and its amount in the Universe has also been well determined by the PLANCK observations [1]. Although dark matter is all pervading in the Universe, their direct evidence in the laboratory is yet to be established mainly because of its non or very weak interaction with other known fundamental particles. The indirect detection of dark matter is based on the principle of detecting Standard Model particles produced due to the self-annihilation of dark matter in a suitable environment. These annihilation products can appear as the excess of the expected flux which could not be explained by other known astrophysical processes. These annihilation products could be $\gamma$ rays, neutrinos, $q \bar{q}$, or lepton antileptons. The satellite-borne experiment AMS-02 (Alpha Magnetic Spectrometer) onboard the International Space Station that looks for

\footnotetext{
*sayan.ghosh@saha.ac.in

†amitdbanik@mail.ccnu.edu.cn

\$ejchun@kias.re.kr

\$debasish.majumdar@saha.ac.in
}

Published by the American Physical Society under the terms of the Creative Commons Attribution 4.0 International license. Further distribution of this work must maintain attribution to the author(s) and the published article's title, journal citation, and DOI. Funded by SCOAP ${ }^{3}$. antimatter in the Universe, has reported an excess of positron fraction beyond the positron energy $10 \mathrm{GeV}$ [2]. The predecessor of the AMS-02 experiment, namely PAMELA [3,4] also reported similar excess of positrons beyond positron energy of $10 \mathrm{GeV}$. The present AMS-02 dataset [5-7] measured up to $800 \mathrm{GeV}$ indicates that the positron fraction decreases with positron energy up to about $10 \mathrm{GeV}$, a phenomenon that can be explained from the behavior of cosmic rays. But beyond $10 \mathrm{GeV}$, the data show a marked increase in the positron fraction that appears to peak around the positron energy of $320 \mathrm{GeV}$. This increase appears to indicate the contribution of additional sources of positrons that may come from dark matter annihilation or decay, and/or from astronomical objects such as pulsars and supernova remnants $[8,9]$, which might be tested by further observations like HAWC [10].

The dark matter interpretation of the positron excess had been pursued earlier by many authors, and after AMS-02 observations, in particular, various scenarios and models have been studied [11-20] either assuming an appropriate boost factor or considering the Sommerfeld effect. Models involving dark matter decay [21-25] as an explanation for the AMS-02 reported positron excess were also addressed by many authors.

In the present work, we propose a specific dark matter model by extending the Standard Model of particle physics by an additional scalar doublet, a singlet fermion, and also a singlet boson. We explicitly work out the phenomenology 
of this model to establish the singlet fermion to be our dark matter candidate. We then calculate the positron excess from the annihilation of this fermionic dark matter after incorporating the light boson mediated Sommerfeld enhancement. The model in fact reduces to a two Higgs doublet model (2HDM) with an additional singlet boson as a portal to the fermion dark matter [26]. The singlet boson, which is taken to be light and responsible for Sommerfeld enhancement, mixes with the pseudoscalar inherently present in the 2HDM. Thus, the dark matter candidate annihilates to the singlet boson that subsequently decays to quarks and leptons.

As is well known, the Sommerfeld enhanced annihilation to quarks and leptons are strongly constrained using the measurements of cosmic microwave background radiation (CMB) anisotropies by PLANCK [1] and the dwarf galaxy results from Fermi-LAT [27]. Such a difficulty can be circumvented by making the singlet boson leptophilic and forbidding the tau final state kinematically. This requires the bosonic portal particle to be very light, which is suited for appropriate Sommerfeld enhancement as was suggested originally in the kinetic portal scenario [28,29]. Thus the dark matter annihilation is supposed to produce four muons, which is shown to provide good fits to the positron excess and spectrum data obtained by the AMS- 02 experiment for the dark matter mass around $1.6 \mathrm{TeV}$ and the annihilation cross section $\langle\sigma v\rangle \sim 2 \times 10^{-23} \mathrm{~cm}^{3} / \mathrm{sec}$ where a local boost factor $\sim 8$ has been adopted. The leptophilic nature can be realized naturally in the lepton-specific $2 \mathrm{HDM}$ with large $\tan \beta$, which is known to accommodate the muon $\mathrm{g}-2$ deviation [30]. The additional light singlet boson can contribute to the muon g-2 as well as other flavor observables. We also analyze, in this work, such an effect to identify the favorable parameter space. Let us remark that observation of gamma rays from the Galactic Center can provide another significant constraint on the dark matter annihilation [31,32]. However, such an observation strongly depends on the dark matter profile [20,33], which have large uncertainties, and thus is not considered in this work.

The paper is organized as follows. In Sec. II we describe the proposed particle dark matter model and discuss particle physics phenomenology of the model, in particular, the impact of the extra singlet boson added to the leptonspecific 2HDM which explains the observed muon g-2. Section III is devoted to the discussion of dark matter relic density, Sommerfeld enhancement and the dark matter mass and couplings relevant for the calculation of the positron excess. The calculations of the positron fraction and positron spectrum, and its variations with positron energy, using the theoretical framework proposed in the work, are performed in Sec. IV. $\chi^{2}$ analyses are also performed in Sec. IV for the AMS-02 experimental results with the calculated results for positron fraction excess and positron spectrum. Finally, some concluding remarks are presented in Sec. V.

\section{THE MODEL FOR LEPTOPHILIC PORTAL}

In this section, we describe the complete particle dark matter model proposed in the present work. We propose a particle physics model for dark matter (DM) by minimally extending the Standard Model (SM) of particle physics with a fermionic singlet $\chi$ and a singlet boson $\phi$, which in this case is a pseudoscalar, and an extra Higgs doublet. The stability of the fermion $\chi$ (the DM candidate) is ensured by imposing a $Z_{2}$ symmetry under which $\chi$ is odd while the SM sector is even. The singlet pseudoscalar acts as a mediator between the DM and the 2HDM having the following interaction [34-42]

$$
\mathcal{L}_{\text {int }}=\phi \bar{\chi}\left(g_{\chi}+i y_{\chi} \gamma^{5}\right) \chi+\phi\left(i b_{\phi} \Phi_{1}^{\dagger} \Phi_{2}+\text { H.c. }\right),
$$

where $b_{\phi}$ is a dimension-one parameter, $g_{\chi}$ and $y_{\chi}$ are the scalar- and pseudoscalarlike couplings between the singlet boson $\phi$ and the DM candidate $\chi$ and $\Phi_{i}(i=1,2)$ are the two Higgs doublet fields. The interaction Lagrangian written in Eq. (1) preserves the $C P$-invariance inherent, at least approximately, in the $2 \mathrm{HDM}$ and the SM sector. However, since the interactions in the dark sector, here involving the fermion $\chi$, are unknown, we include a $C P$ violating interaction between the pseudoscalar $\phi$ and the DM candidate $\chi$. This $C P$-violating coupling $\left(g_{\chi}\right)$ originally is a property of the dark sector. In general the Lagrangian $\mathcal{L}_{\text {int }}$ also contains terms to the tune of $\kappa\left(\phi^{2} \Phi_{1}^{\dagger} \Phi_{2}+\right.$ H.c. $)$ and therefore would add to the scalar trilinear couplings discussed in subsequent sections. It will also be seen in later sections that these scalar trilinear couplings are bounded to small values and therefore for small enough values of $\kappa$, the contribution to the parameter space due to this additional term in the Lagrangian can be neglected.

In the 2HDM sector, we have two charged Higgs fields $\left(H^{ \pm}\right)$, two $C P$-even scalar fields $(h, H)$, one $C P$-odd scalar $\left(A_{0}\right)$ and three Goldstone bosons $\left(G^{ \pm}, G^{0}\right)$. The Higgs doublets, $\Phi_{1}$ and $\Phi_{2}$, are written in the form [43,44]

$$
\begin{aligned}
& \Phi_{1}=\left(\begin{array}{c}
c_{\beta} G^{+}-s_{\beta} H^{+} \\
\frac{1}{\sqrt{2}}\left(v_{1}+c_{\alpha} H-s_{\alpha} h+i c_{\beta} G-i s_{\beta} A_{0}\right)
\end{array}\right), \\
& \Phi_{2}=\left(\begin{array}{c}
s_{\beta} G^{+}+c_{\beta} H^{+} \\
\frac{1}{\sqrt{2}}\left(v_{2}+s_{\alpha} H+c_{\alpha} h+i s_{\beta} G+i c_{\beta} A_{0}\right)
\end{array}\right) .
\end{aligned}
$$

Here $\alpha$ is the mixing angle between the two $C P$-even scalars and $c_{x}$ and $s_{x}(x=\alpha, \beta)$ represent $\cos x$ and $\sin x$, respectively. We consider the lepton-specific 2HDM where the leptons acquire their masses from their Yukawa couplings with the $\Phi_{1}$ doublet, while quarks get their masses due to their Yukawa couplings with the $\Phi_{2}$ doublet. Then the Yukawa couplings of the physical Higgs fields can be written as 


$$
\begin{aligned}
-\mathcal{L}_{\text {Yuk }}= & \sum_{f=u, d, l} \frac{m_{f}}{v}\left(y_{f}^{h} h \bar{f} f+y_{f}^{H} H \bar{f} f-i y_{f}^{A_{0}} A_{0} \bar{f} \gamma^{5} f\right) \\
& +\left[\sqrt{2} V_{u d} H^{+} \bar{u}\left(\frac{m_{u}}{v} y_{u}^{A_{0}} P_{L}+\frac{m_{d}}{v} y_{d}^{A_{0}} P_{R}\right) d+\text { H.c. }\right] \\
& +\left[\sqrt{2} \frac{m_{l}}{v} y_{l}^{A_{0}} H^{+} \bar{\nu} P_{R} l+\text { H.c. }\right]
\end{aligned}
$$

where $y_{f}^{h, H, A_{0}}$ are the normalized Yukawa couplings of fermions and $v=\sqrt{v_{1}^{2}+v_{2}^{2}}=246 \mathrm{GeV}$ is the total Higgs vacuum expectation value. Here the $C P$-even scalar $h$ denotes the SM-like Higgs boson with mass $m_{h}=$ $125 \mathrm{GeV}$, and $H$ is the additional Higgs boson with heavier mass $m_{H}$. The normalized Yukawa couplings for only the $\mathrm{CP}$-odd Higgs boson are given as

$$
y_{u}^{A_{0}}=\cot \beta, \quad y_{d}^{A_{0}}=-\cot \beta, \quad y_{l}^{A_{0}}=\tan \beta,
$$

where $\tan \beta \equiv v_{2} / v_{1}$.

Notice that we have introduced only the pseudoscalar coupling between the singlet boson $\phi$ and $\Phi_{1,2}$, in order to preserve the $C P$ invariance in the $2 \mathrm{HDM}$ sector. As a result, $A_{0}$ mixes with $\phi$ and the mass-squared matrix can be written as

$$
\mathcal{M}_{A}^{2}=\left(\begin{array}{cc}
m_{A_{0}}^{2} & b_{\phi} v \\
b_{\phi} v & m_{\phi}^{2}
\end{array}\right) .
$$

Diagonalization of $\mathcal{M}_{A}^{2}$ by the rotation of the state $\left(\begin{array}{c}A_{0} \\ \phi\end{array}\right)$ as

$$
\left(\begin{array}{l}
A \\
a
\end{array}\right)=\left(\begin{array}{cc}
\cos \theta & \sin \theta \\
-\sin \theta & \cos \theta
\end{array}\right)\left(\begin{array}{c}
A_{0} \\
\phi
\end{array}\right)
$$

leads to the relation

$$
b_{\phi}=\frac{1}{2 v}\left(m_{A}^{2}-m_{a}^{2}\right) \sin 2 \theta,
$$

where $m_{A, a}^{2}$ are the eigenvalues of $\mathcal{M}_{A}^{2}$ for the rotated eigenstate $\left(\begin{array}{c}A \\ a\end{array}\right)$.

In terms of the physical $C P$-odd eigenstates $A$ and $a$, the interaction Lagrangian $\mathcal{L}_{\text {int }}$ now takes the form

$$
\mathcal{L}_{\text {int }} \supset(A \sin \theta+a \cos \theta) \bar{\chi}\left(g_{\chi}+i y_{\chi} \gamma^{5}\right) \chi .
$$

Accordingly, the Yukawa Lagrangian in Eq. (4) modifies as

$$
-\mathcal{L}_{\mathrm{Yuk}} \supset-i \sum_{f=u, d, l} \frac{m_{f}}{v} y_{f}^{A_{0}}(\cos \theta A-\sin \theta a) \bar{f} \gamma^{5} f .
$$

From Eqs. (9)-(10), the couplings of the physical states $a$ and $A$ to quarks, leptons, and DM fermions can be written as

$$
\begin{aligned}
y_{l}^{a} & =-\tan \beta \sin \theta, & y_{l}^{A} & =\tan \beta \cos \theta, \\
y_{u}^{a} & =-\cot \beta \sin \theta, & y_{u}^{A} & =\cot \beta \cos \theta, \\
y_{d}^{a} & =\cot \beta \sin \theta, & y_{d}^{A} & =-\cot \beta \cos \theta, \\
g_{a \bar{\chi} \chi} & =g_{\chi} \cos \theta, & g_{A \bar{\chi} \chi} & =g_{\chi} \sin \theta, \\
y_{a \bar{\chi} \chi} & =y_{\chi} \cos \theta, & y_{A \bar{\chi} \chi} & =y_{\chi} \sin \theta .
\end{aligned}
$$

Having assumed $C P$ invariance in the $2 \mathrm{HDM}$ sector, the physical $C P$-odd state $a$ (and also $A$ ) does not have any couplings of the type $a Z Z, a W^{+} W^{-}$, or $a h h$.

\section{A. LHC constraints}

The lepton-specific 2HDM becomes leptophilic at large $\tan \beta$ (called Leptophilic-2HDM or L2HDM) and thus easily avoids constraints from hadronic processes. The extra Higgs bosons $a, A, H$, and $H^{ \pm}$in the present model can hardly be produced only through the quark couplings at the LHC. However, one can still probe their existence through the Drell-Yan process [45] or the Standard Model Higgs decays, $h \rightarrow a a, a A, A A$ [46] if allowed kinematically. In the present work, the pseudoscalars $a$ and $A$ are required to be very light $m_{a} \ll m_{A}<m_{h} / 2$ and thus their production through $h \rightarrow \phi \phi^{\prime}\left(\phi, \phi^{\prime}=a, A\right)$ may be observed if the corresponding coupling $g_{h \phi \phi^{\prime}}$ (see [47] for the full expression) is large enough. The decay width for such a process is given by

$$
\Gamma_{h \rightarrow \phi \phi^{\prime}}=S \frac{g_{h \phi \phi^{\prime}}^{2}}{32 \pi m_{h}} \sqrt{1-\frac{\left(m_{\phi}+m_{\phi^{\prime}}\right)^{2}}{m_{h}^{2}}},
$$

where $S=2$ for $\phi \neq \phi^{\prime}$. Indeed such channels are examined by the LHC experiments. Considering the $2 \mu 2 \tau$ and $4 \mu$ searches at CMS [48,49], we obtain the following limits

$$
\begin{gathered}
g_{h A A} / v \lesssim 0.008 \text { for } m_{A}=15-62 \mathrm{GeV}, \\
g_{\text {haa }} / v \lesssim 0.003 \text { for } m_{a}=0.5-3.5 \mathrm{GeV},
\end{gathered}
$$

which are applicable to the mass ranges under consideration.

\section{B. Flavor constraints}

As is well known, the L2HDM can explain the muon g-2 anomaly due to the presence of a light pseudoscalar $A$ $[30,50]$ whose parameter space is constrained by the $B_{s} \rightarrow \mu^{+} \mu^{-}$decay [51] and lepton universality conditions in $Z \rightarrow l l$ and $\tau / \mu$ decays [52]. The presence of another (lighter) pseudoscalar $a$ can modify the previous results and puts a limit in its parameter space. Generalizing the calculations in [53], we update the conventional parameter region of $\left(m_{A}, \tan \beta\right)$ favored by the recent measurement of $a_{\mu}=(g-2)_{\mu} / 2$ at FNAL [54]: 


$$
a_{\mu}^{\text {Exp }}-a_{\mu}^{\mathrm{Th}}=251(59) \times 10^{-11}
$$

and examine the impact of $a$. In Fig. 1, we show the region of parameter space consistent with the $2 \sigma$ range of the muon g-2 data when $m_{H^{ \pm}}=m_{H}=300 \mathrm{GeV}$ is adopted. The region inside the solid blue contours is for the pure L2HDM. In this case there is negligible mixing with $a$ signifying $\sin \theta \rightarrow 0$. When the mixing is turned on, the light pseudoscalar gives a negative contribution to the muon $\mathrm{g}-2$ and thus large $\tan \beta$ and smaller $m_{A}$ are required to explain the muon g- 2 as shown by the dashed blue lines in both the left and right panels. This leads to the upper limit on the mixing angle

$$
\sin \theta=0.12-0.3,
$$

for the preferred range of $m_{a}\left(m_{a}=1.5-3.5 \mathrm{GeV}\right)$ as will be discussed in the following sections. A light pseudoscalar can serve well as a mediator of $B_{s}$ decays to a pair of leptons. Its impact especially on the $B_{s} \rightarrow \mu^{+} \mu^{-}$decay channel can be sizable due to its "tree-level" exchange accompanied by loop-level flavor changing neutral currents. Recently, the LHCb collaboration [55] reported the $B_{s} \rightarrow \mu^{+} \mu^{-}$branching ratio to be $\operatorname{Br}\left(B_{s} \rightarrow \mu^{+} \mu^{-}\right)=\left(3.0 \pm 0.6_{-0.2}^{+0.3}\right) \times 10^{-9}$, which is largely consistent with the Standard Model prediction. We do not attempt to revise the analysis accordingly but quote the previous results constraining the mediator mass $m_{A} \gtrsim 10 \mathrm{GeV}$ [51], which can be translated to $m_{a} \gtrsim 10 \sin \theta \mathrm{GeV}$.

\section{Searches for a light leptophilic boson}

There have been attempts to search for new light particles that have been predicted to couple to the Standard Model in various theoretical frameworks [56].

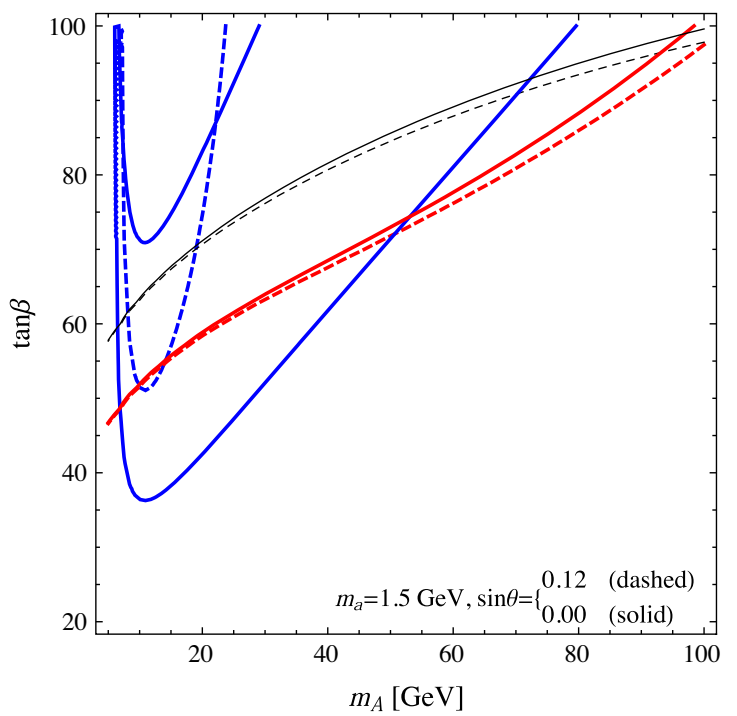

Extending previous studies on leptophilic bosons [57,58], we further obtain the current constraints and future sensitivity on the parameter space of our leptophilic pseudoscalar. Its lepton coupling proportional to $\sim \theta \tan \beta$ can be probed directly by lepton processes at ORSAY [59], E137 [60], and $B A B A R$ [61], as well as the future experiments such as NA64-muon [62], SEAQUEST [63], and lepton colliders $[64,65]$. The current and future search limits are presented by solid lines in Fig. 2. The three shaded areas are excluded by ORSAY, E137, and BABAR data, respectively. As the quark coupling is suppressed by $1 / \tan ^{2} \beta$ compared to the lepton coupling, hadronic processes are inefficient to probe such a leptophilic boson. We show the current and future limits by three dotted contours for LHCb [66], BELLE II [67], and SHIP [68] (overlapping almost with MATHUSLA [69]). For this we considered the dominant process of $b \rightarrow s a(\mu \mu)$ fixing $\tan \beta=40$. The yellow band shows our preferred region of $m_{a} \approx 1.5-3.5 \mathrm{GeV}$.

\section{DARK MATTER PHENOMENOLOGY}

The interaction Lagrangian for the dark matter candidate and its portal in the framework of the present model (see Sec. II), relevant for our analysis, can be written as

$$
\mathcal{L}=a \bar{\chi}\left(g_{a \chi \chi}+i y_{a \chi \chi} \gamma^{5}\right) \chi+i a \sum_{f} \frac{m_{l}}{v} y_{l}^{a} \bar{l} \gamma^{5} l
$$

where $g_{a \chi \chi}=g_{\chi} \cos \theta, y_{a \chi \chi}=y_{\chi} \cos \theta$, and $y_{l}^{a}=\tan \beta \sin \theta$ for $l=e, \mu, \tau$. Note that we have omitted the quark coupling $y_{q}^{a}=\sin \theta / \tan \beta=y_{l}^{a} / \tan ^{2} \beta$, which is highly suppressed for large $\tan \beta$ under consideration. To study the DM properties, we will restrict ourselves to the small

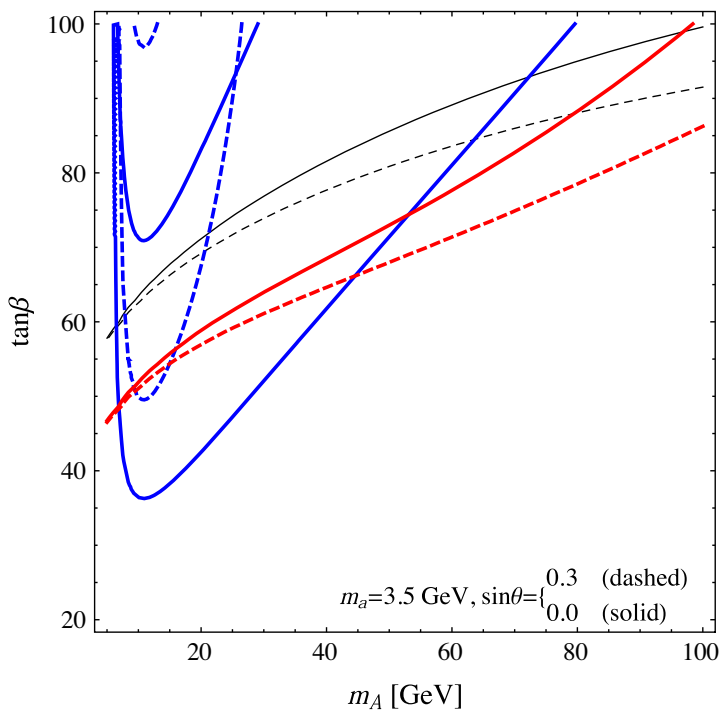

FIG. 1. The combined $2 \sigma$ allowed parameter space in the $\tan \beta$ vs $m_{A}$ plane with $m_{a}=1.5 \mathrm{GeV}$ (left panel) and $m_{a}=3.5 \mathrm{GeV}$ (right panel) from the $a_{\mu}$ results (blue) and upper limits from the lepton-flavor universality bounds in the $Z$ (red) and $\tau / \mu$ (black) decays. We have assumed $m_{H}=m_{H^{ \pm}}=300 \mathrm{GeV}$. 


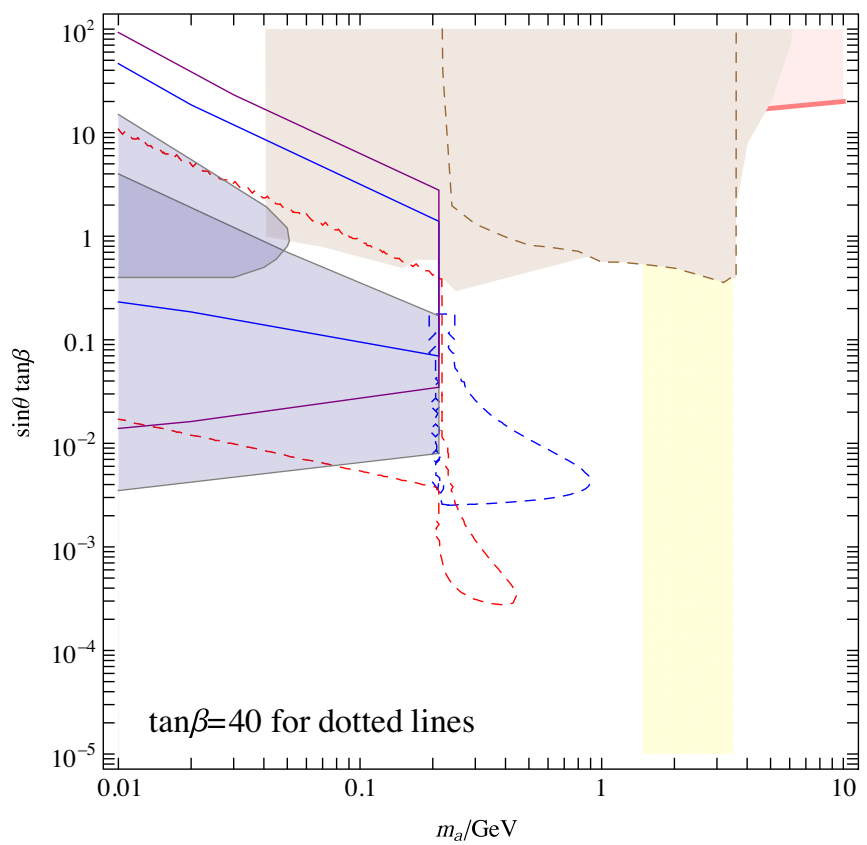

FIG. 2. The shaded regions are excluded by $B A B A R$ (light brown) and ORSAY and E137 (light blue), and the solid contours are future sensitivities at NA64-muon (purple), SEAQUEST (blue), and lepton colliders (pink on the right upper corner). The shaded region inside the dotted line is excluded by $\mathrm{LHCb}$ (light brown), and the other dotted contours are the future sensitivity at BELLE II (blue) and SHIP/MATHUSLA (red).

mixing limit $\sin \theta \approx 0$ for which we can take the approximation $g_{a \chi \chi} \approx g_{\chi}$ and $y_{a \chi \chi} \approx y_{\chi}$.

\section{A. Dark matter annihilation and relic density}

The Feynman diagrams for the main DM annihilation channels in this framework are shown in Fig. 3. The total annihilation cross section is given as

$$
\begin{aligned}
\left\langle\sigma v_{\text {rel }}\right\rangle_{\bar{\chi} \chi \rightarrow a a} \simeq & {\left[\frac{g_{\chi}^{2} y_{\chi}^{2}}{8 \pi m_{\chi}^{2}}+\frac{3 g_{\chi}^{4}}{64 \pi m_{\chi}^{2}}\left\langle v^{2}\right\rangle+\frac{y_{\chi}^{4}}{384 \pi m_{\chi}^{2}}\left\langle v^{2}\right\rangle\right] } \\
& \times\left(1-\frac{m_{a}^{2}}{m_{\chi}^{2}}\right)^{1 / 2} .
\end{aligned}
$$

It can be seen that the annihilation due to the second and third $t$-channel processes suffer a $p$-wave suppression of

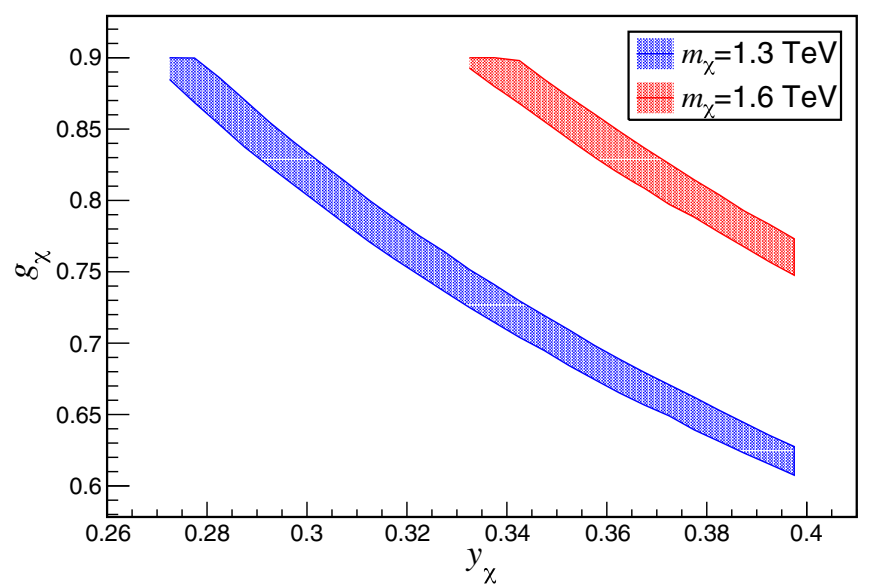

FIG. 4. The allowed parameter region in the $g_{\chi}$ vs $y_{\chi}$ plane that satisfies the PLANCK observed DM relic density for dark matter masses of 1.3 and $1.6 \mathrm{TeV}$.

order $\mathcal{O}\left(v_{\text {rel }}^{2}\right)$ [70,71]. Although at the freeze-out temperature of $\chi\left(v_{\text {rel }} \sim 0.3\right)$ the contribution to the DM relic density from these processes cannot be neglected, they are highly suppressed at the present epoch $\left(v_{\text {rel }} \sim 10^{-3}\right)$. As a result, observable signals will appear only through the first process, which will be responsible for the positron excess.

Following the standard calculation of the DM freeze-out process [72], one can constrain the parameters $m_{\chi}, g_{\chi}$, and $y_{\chi}$, leading to the observed DM relic density, which requires $\left\langle\sigma v_{\text {rel }}\right\rangle \approx 3 \times 10^{-26} \mathrm{~cm}^{3} / \mathrm{sec}$. In Fig. 4 we show the variation of the scalar $g_{\chi}$ as a function of pseudoscalarlike coupling $y_{\chi}$, for DM masses 1.3 and $1.6 \mathrm{TeV}$, respectively, in order to agree with the DM relic density obtained from the PLANCK experimental results. Three sets of parameters designated by sets I, II and III are chosen from the allowed parameter space with $y_{\chi}=0.4$. These sets are given in Table I. These will be used to obtain appropriate Sommerfeld enhancement factors and then performing the $\chi^{2}$ fit to the positron excess in the following sections.

\section{B. Direct detection of dark matter}

In the present work, with a leptophilic pseudoscalar mediator to quarks, the tree-level process for the DMnucleon scattering is spin dependent and its cross section, being proportional to $\vec{q}^{2} / m_{\chi}^{2}$ ( $\vec{q}$ is a momentum transfer)
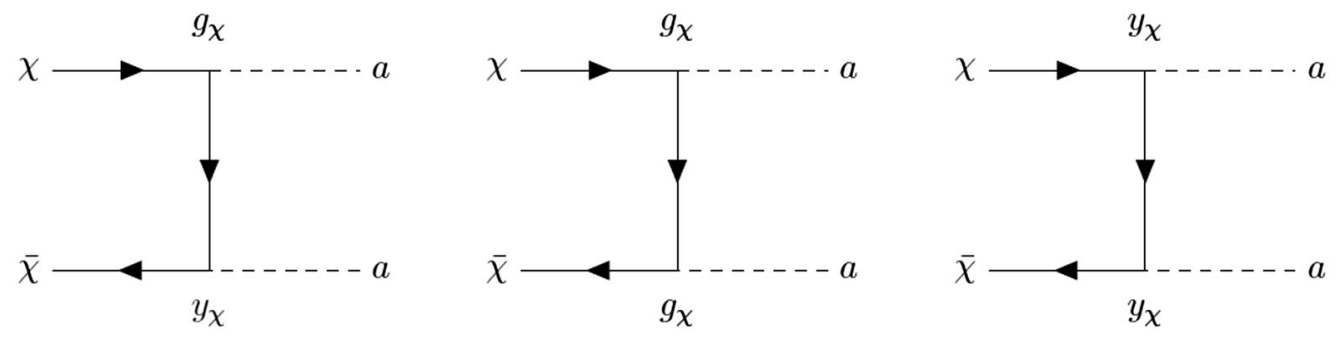

FIG. 3. The diagrams for DM annihilation into $a a$. 
TABLE I. The representative sets of parameters leading to the observed DM relic density and appropriate Sommerfeld enhancement.

\begin{tabular}{lcccc}
\hline \hline & $m_{\chi}(\mathrm{TeV})$ & $m_{a}(\mathrm{GeV})$ & $g_{\chi}$ & $y_{\chi}$ \\
\hline Set I & 1.3 & 1.97 & 0.60 & 0.4 \\
Set II & 1.6 & 3.42 & 0.75 & 0.4 \\
Set III & 1.9 & 3.45 & 0.88 & 0.4 \\
\hline \hline
\end{tabular}

and the quantity $\sin ^{2} \theta / \tan ^{2} \beta$, is too much suppressed to be observable in the foreseeable future. There are also oneloop processes through the box and triangle diagrams in Fig. 5 that give rise to spin-independent DM-nucleon scattering $[36,47]$. In our case, however, the box diagram is proportional to $\sin ^{2} \theta / \tan ^{2} \beta$ and the triangle diagram involves the small couplings $g_{\text {haa }} / v \lesssim 0.003$ and $y_{H q q} \approx$ $\frac{m_{q}}{v \tan \beta}$ in amplitudes. As a result, we find the corresponding cross sections are below the neutrino forward scattering cross section limit for $m_{\chi} \sim 1 \mathrm{TeV}$. Therefore, the DM candidate in the present model may not be probed by direct detection experiments.

\section{Sommerfeld enhancement}

In the early Universe, at around the freeze-out temperature of the thermal relics, the relative velocity of DM particles was relativistic with $v_{\text {rel }} \sim 0.3$. The annihilation rate and the relic abundance were thus determined by this relative velocity. As discussed above, the required annihilation rate for DM particles to reproduce the observed relic abundance is $\left\langle\sigma v_{\text {rel }}\right\rangle \simeq 3.0 \times 10^{-26} \mathrm{~cm}^{3} / \mathrm{sec}$. In the present Universe, however, the DM particles are highly nonrelativistic with $v_{\text {rel }} \sim 10^{-3}$, and thus the DM annihilation can get enhanced considerably by the Sommerfeld effect [73]. The thermally averaged annihilation cross section in the nonrelativistic limit can be written as

$$
\left\langle\sigma v_{\text {rel }}\right\rangle=\sqrt{\frac{2}{\pi}} \frac{1}{v_{0}^{3}} \int v_{\text {rel }}^{2} e^{-\left(v_{\text {rel }}^{2} / 2 v_{0}^{2}\right)} \sigma v_{\text {rel }} d v_{\text {rel }},
$$

where $v_{0} \simeq 220 \mathrm{~km} / \mathrm{sec}$ is the most probable velocity of DM in the galaxy. One can also write the most probable velocity as

$$
v_{0}=\sqrt{\frac{2}{x_{\chi}}},
$$

with $x_{\chi}=m_{\chi} / T_{\chi}, T_{\chi}$ being the temperature of DM today. We can also write the Sommerfeld enhanced annihilation rate $\sigma v_{\text {rel }}$ as $\left(\sigma v_{\text {rel }}\right)_{0} S_{0}\left(v_{\text {rel }}\right)$, where $S_{0}\left(v_{\text {rel }}\right)$ is the velocitydependent Sommerfeld enhancement factor. Then, one obtains [74]

$$
\begin{aligned}
\left\langle\sigma v_{\text {rel }}\right\rangle & =\frac{x_{\chi}^{3 / 2}}{2 \sqrt{\pi}} \int_{0}^{v_{\mathrm{esc}}}\left(\sigma v_{\mathrm{rel}}\right) v_{\mathrm{rel}}^{2} e^{-\left(x_{\chi} v_{\mathrm{rel}}^{2} / 4\right)} d v_{\mathrm{rel}}, \\
& =\left(\sigma v_{\mathrm{rel}}\right)_{0}\langle S\rangle
\end{aligned}
$$

where $v_{\text {esc }} \simeq 550 \mathrm{~km} / \mathrm{sec}$ is the escape velocity for DM in the DM halo of our galaxy and

$$
\langle S\rangle=\frac{x_{\chi}^{3 / 2}}{2 \sqrt{\pi}} \int_{0}^{v_{\mathrm{esc}}} S_{0}\left(v_{\mathrm{rel}}\right) v_{\mathrm{rel}}^{2} e^{-\left(x_{\chi} v_{\mathrm{rel}}^{2} / 4\right)} d v_{\mathrm{rel}}
$$

stands for the thermally averaged Sommerfeld enhancement.

In the present framework, the DM interacts with the light $C P$-odd particle $a$ through both the scalar and pseudoscalar couplings. In Sec. III A, we have chosen the scalar coupling $g_{\chi}$ to be greater than the pseudoscalar coupling $y_{\chi}$ so that the $a-\chi$ scalar interaction is more effective and relevant for the Sommerfeld enhancement [75]. In order to calculate the Sommerfeld factor, we rely on the analytic formula given by [76,77]

$$
S_{0}=\frac{\pi}{\epsilon_{v}} \frac{\sinh \left(\frac{2 \pi \epsilon_{v}}{\pi^{2} \epsilon_{a} / 6}\right)}{\cosh \left(\frac{2 \pi \epsilon_{v}}{\pi^{2} \epsilon_{a} / 6}\right)-\cos \left(2 \pi \sqrt{\frac{1}{\pi^{2} \epsilon_{a} / 6}-\frac{\epsilon_{v}^{2}}{\left(\pi^{2} \epsilon_{a} / 6\right)^{2}}}\right)},
$$

where $\epsilon_{v}=v_{\text {rel }} / \alpha_{\chi}$ and $\epsilon_{a}=m_{a} / \alpha_{\chi} m_{\chi}$. This analytical solution is known to match well, within $10 \%$ variation, with the numerical solutions [74]. It is now crucial to require that $\epsilon_{v}<\epsilon_{\alpha}$, saturating the velocity dependence, and to obtain the Sommerfeld-enchanced annihilation rate around $10^{-24} \mathrm{~cm}^{3} / \mathrm{sec}$ (that is, $\langle S\rangle \sim 100$ ), which is allowed by the $\mathrm{CMB}$ limit for the annihilation process $\chi \chi \rightarrow a a \rightarrow 4 \mu$. Thus, we are forced to be in a narrow range of the light boson mass, $1.5 \mathrm{GeV}<m_{a}<3.5 \mathrm{GeV}$, as
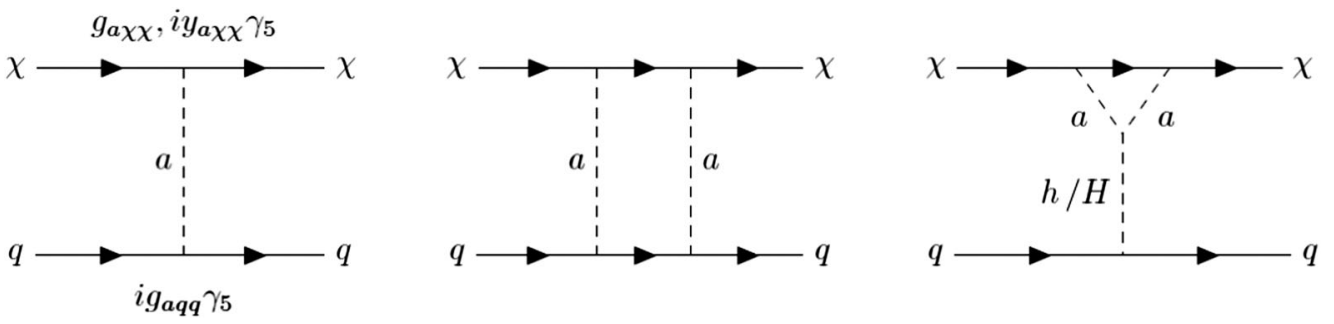

FIG. 5. The dominant tree and loop (box and triangle) diagrams for DM direct detection. The coupling are given by $g_{a \chi \chi}=g_{\chi} \cos \theta$, $y_{a \chi \chi}=y_{\chi} \cos \theta$, and $g_{a q q}=\left(m_{q} / v\right) \sin \theta / \tan \beta$. 
shown in Table I. We will see that the DM mass needs to be in the range, $1.3 \mathrm{TeV} \leq m_{\chi} \leq 1.9 \mathrm{TeV}$, to fit the positron excess with an additional boost factor of $B \sim 8$.

\section{POSITRON EXCESSES}

Having set all the relevant ingredients for the DM annihilation, we calculate first the positron flux from DM annihilation and finally the positron fraction resulting from the dominant channel $\bar{\chi} \chi \rightarrow a a \rightarrow 4 \mu$. The thermally averaged cross section of this channel in our galaxy is given by

$$
\left\langle\sigma v_{\text {rel }}\right\rangle_{\bar{\chi} \chi \rightarrow a a} \approx \frac{g_{\chi}^{2} y_{\chi}^{2}}{8 \pi m_{\chi}^{2}}\langle S\rangle .
$$

In this work, we have chosen $m_{a}<2 m_{\tau}$, and as a result the $a \rightarrow \tau^{+} \tau^{-}$is kinematically forbidden. Therefore $a$ will readily decay to $2 \mu$, which can finally produce positrons. The positron fraction as a result of DM annihilations, per unit energy, is given by [78]

$$
\frac{d \Phi_{e^{+}}}{d E}(t, \vec{x}, E)=v_{e^{+}} \frac{f}{4 \pi} \mathrm{GeV}^{-1} \mathrm{~cm}^{-2} \mathrm{~s}^{-1} \mathrm{sr}^{-1},
$$

where $v_{e^{+}}$is the velocity of positron, and the quantity $f=$ $\frac{d N_{e^{+}}}{d E}$ represents the positron number density per unit energy. The latter follows the diffusion equation $[79,80]$

$\frac{\partial f}{\partial t}-\nabla(\mathcal{K}(E, \vec{x}) \nabla f)-\frac{\partial}{\partial E}(b(E, \vec{x}) f)=Q(E, \vec{x})$,

where $\mathcal{K}(E, \vec{x})$ and $b(E, \vec{x})$ are the diffusion coefficient function and energy loss coefficient function, respectively. The source term $Q$ in Eq. (25) is given by [33]

$$
Q=\frac{1}{2}\left(\frac{\rho_{\mathrm{DM}}}{m_{\mathrm{DM}}}\right)^{2} f_{i n j}^{\mathrm{ann}},
$$

where the quantity $f_{i n j}^{\text {ann }}=\sum_{k}\langle\sigma v\rangle_{k} \frac{d N_{e^{+}}^{k}}{d E}$. Here $k$ represents all annihilation channels with $e^{+}$in the final state, $\rho_{\mathrm{DM}}$ and $m_{\mathrm{DM}}$ represent the DM density and mass, respectively, and $\frac{d N_{e^{+}}}{d E}$ is the positron spectrum. The differential positron flux at Earth with positron energy $E$ from DM annihilation can be written as $[33,78]$

$$
\begin{aligned}
\frac{d \Phi_{e^{+}}}{d E}\left(E, \vec{r}_{\odot}\right)= & B \frac{v_{e^{+}}}{4 \pi b(E, \vec{x})} \frac{1}{2}\left(\frac{\rho_{\odot}}{m_{\mathrm{DM}}}\right)^{2} \sum_{k}\langle\sigma v\rangle_{k} \\
& \times \int_{E}^{m_{\mathrm{DM}}} d E_{s} \frac{d N_{e^{+}}^{k}}{d E}\left(E_{s}\right) I\left(E, E_{s}, \vec{r}_{\odot}\right),
\end{aligned}
$$

where $E_{s}$ stands for the positron energy at production (source) and $I\left(E, E_{s}, \vec{r}_{\odot}\right)$ is the generalized halo function at Earth, which is in fact the Green function from a source with energy $E_{s}$. In the above, the local DM density is considered to be $\rho_{\odot} \sim 0.3 \mathrm{GeV} / \mathrm{cm}^{3}$, and $B$ represents the cosmological boost factor which accounts for DM clumping. The positron flux at Earth, from the annihilation of the DM candidate proposed in this work, is calculated by using the publicly available code PPPC4DMID $[33,81]$. The boost factor $B$ depends on the energy $E$ of the positron [82-84] with a value that lies between 1-20 [85] depending on the energy. A reasonable value of $B \sim 10$ [78,86]. The positron fraction as a result of DM annihilations in this work is given by [13]

$$
F_{e^{+}}=\frac{\Phi_{e^{+}}^{\mathrm{sig}}+\Phi_{e^{+}}^{\mathrm{bkg}}}{2 \Phi_{e^{+}}^{\mathrm{sig}}+\Phi_{e^{+}}^{\mathrm{bkg}}+\Phi_{e^{-}}^{\mathrm{bkg}}},
$$

where $\Phi_{e^{+}}^{\mathrm{sig}}$ and $\Phi_{e^{ \pm}}^{\mathrm{bkg}}$ denote the flux of the positrons from DM annihilation and background cosmic ray flux, respectively, and it has been assumed that the positron and electron fluxes produced as a result of DM annihilation are same. The positron background has been computed by adopting a parametrization of the background as [6]

$$
\Phi_{e^{+}}^{\mathrm{bkg}}(E)=\frac{E^{2}}{\hat{E}^{2}} C_{d}\left(\hat{E} / E_{1}\right)^{\gamma_{d}},
$$

where $\hat{E}=E+\phi_{e^{+}}$, with $\phi_{e^{+}}$accounting for the solar effects. In Eq. (29) above, the parameters $E_{1}=7.0 \mathrm{GeV}$, $\gamma_{d}=-3.6, \quad C_{d}=6.42 \times 10^{-2} \mathrm{GeV}^{-1} \mathrm{~m}^{-1} \mathrm{~s}^{-1} \mathrm{sr}^{-1}, \quad$ and $\phi_{e^{+}}=0.869 \mathrm{GeV}$ [14], and

$$
\Phi_{e^{-}}^{\mathrm{bkg}}(E)=C_{e}\left(E / E_{1 e}\right)^{\gamma_{e}},
$$

gives the background parametrization for electrons [7], where $E_{1 e}=42.01 \mathrm{GeV}, \gamma_{e}=-3.3$, and $C_{e}=2.1 \times$ $10^{-3} \mathrm{GeV}^{-1} \mathrm{~m}^{-1} \mathrm{~s}^{-1} \mathrm{sr}^{-1}$.

TABLE II. The values of the DM mass $m_{\chi}$, Sommerfeld enhancement $\langle S\rangle$, the boost factor $B$, and the annihilation cross sections including the Sommerfeld effect and then boosted by the local factor $B$. The values of the other parameters used in the calculation are given in Table I.

\begin{tabular}{lcrccc}
\hline \hline & $m_{\chi}(\mathrm{TeV})$ & $\langle S\rangle$ & $\left\langle\sigma v_{\text {rel }}\right\rangle\left(\mathrm{cm}^{3} / \mathrm{sec}\right)$ & $B$ & $B\left\langle\sigma v_{\text {rel }}\right\rangle\left(\mathrm{cm}^{3} / \mathrm{sec}\right)$ \\
\hline BP1 & 1.3 & 80 & $1.30 \times 10^{-24}$ & 7.02 & $0.91 \times 10^{-23}$ \\
BP2 & 1.6 & 98 & $1.59 \times 10^{-24}$ & 8.24 & $1.31 \times 10^{-23}$ \\
BP3 & 1.9 & 148 & $2.39 \times 10^{-24}$ & 7.38 & $1.77 \times 10^{-23}$ \\
\hline \hline
\end{tabular}



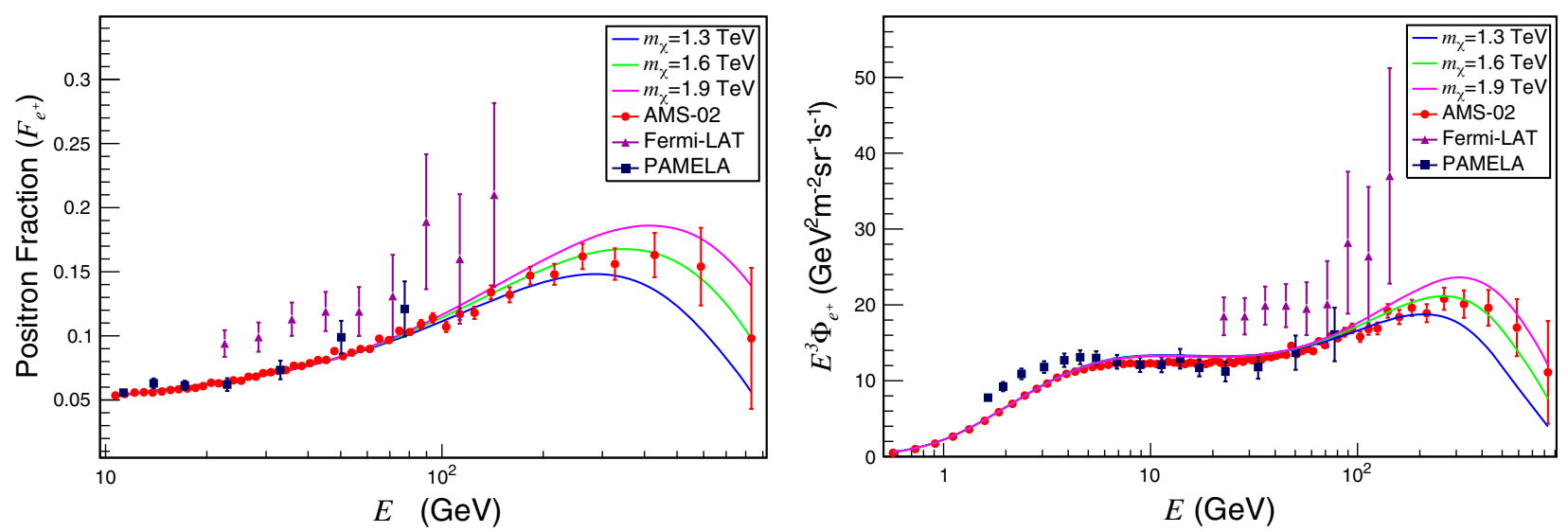

FIG. 6. Left panel: the positron fraction obtained from the DM annihilation for the different benchmark points tabulated in Table II. The experimental data as reported by AMS-02, Fermi-LAT, and PAMELA are also shown. Right panel: the comparison of the positron flux using the best fit parameters obtained from fitting the AMS-02 observed positron fraction, with the experimental data published by the three experiments.

Following the formalism given above, we compute the positron fraction as obtained from the DM annihilation for the DM candidate proposed in this work for various benchmark points. A $\chi^{2}$ analysis was performed for the data and with the theoretical formalism described in this work. The boost factor $B$ is the free parameter to be evaluated by $\chi^{2}$ minimization. The best-fit values of the boost factor $B$ (parameter) along with the values of the DM mass $m_{\chi}$, the DM-pseudoscalar coupling $y_{\chi}$ and the Sommerfeld enhanced cross section for the $\bar{\chi} \chi \rightarrow a a$ channel for the different benchmark points are given in Table II. The final computed positron-fraction results are furnished in Fig. 6 (left panel). Also shown in Fig. 6 (left panel) is the positron fraction data as observed by the AMS02 [7], Fermi-LAT [87], and PAMELA [88] experiments. The right panel of Fig. 6 shows the comparison of the positron flux as computed from the present DM model for various benchmark points using the best fit parameters obtained by fitting the positron-fraction data of AMS-02, with that as reported by the AMS-02 [6], Fermi-LAT, and PAMELA experiments. For this plot, we consider the diffuse flux to be the same as the positron background model laid out in Eq. (29). It can be seen from Fig. 6 (left panel) that the DM model considered in this work fits well for the excess in positron fraction in the energy region $E \geq 10 \mathrm{GeV}$. Therefore the observational results of AMS02 as presented in [7] can be explained by the annihilation of a fermionic DM candidate preferably for the DM mass of $1.6 \mathrm{TeV}$ through a $\mu$-philic pseudoscalar portal as proposed in this work. It can also be seen that the positron spectrum [6] could be simultaneously fitted by using the same parameter set as shown in the right panel. We remark that the fit to the positron spectrum is marginally worse than that to the positron fraction. This discrepancy can be relaxed by choosing an appropriate propagation model of cosmic rays [19].
In addition to the AMS-02 experimental data for positron flux and fraction, we also study the comparison of our theoretical prediction of $e^{+}+e^{-}$flux with the measurements of CALET [89] and DAMPE [90] experiments as well as with that of AMS-02 [7]. We present our results in Fig. 7. The total $e^{+}+e^{-}$flux, from our theoretical framework, has been computed using the best fit parameters, tabulated in Table II, obtained while fitting the AMS-02 positron fraction results. It can be seen that the present DM model can well explain, simultaneously, the positron fraction and positron flux data of the AMS-02 experiment as well as the total cosmic ray flux observations of CALET. We find that, with the same model parameter values, shown in Table II, the fit to the DAMPE results do not compare well to that of AMS-02 and CALET. However, with the present DM model, choosing a different set of values for the parameters, one can also obtain a very good fit to the DAMPE experimental $e^{+}+e^{-}$ data. For instance, considering a DM mass of $3.0 \mathrm{TeV}$

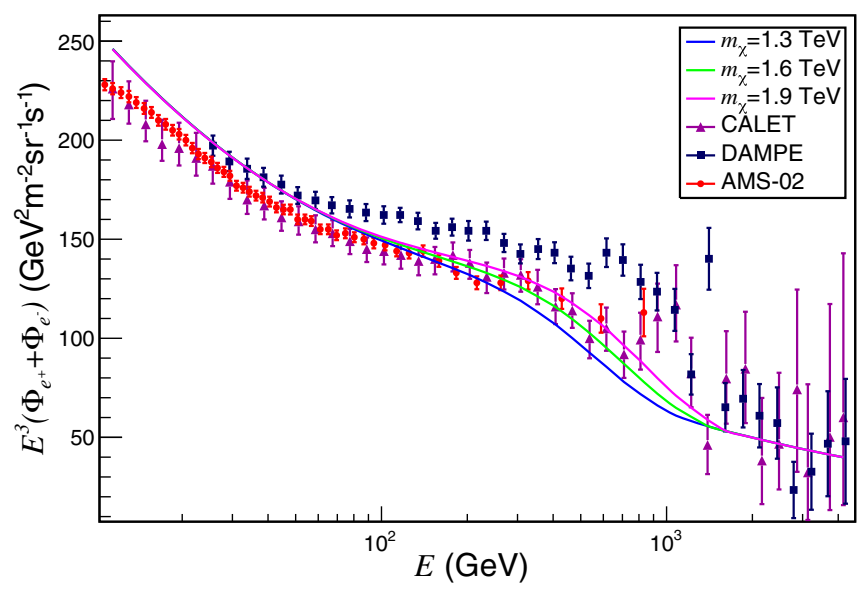

FIG. 7. Comparison of the total $e^{+}+e^{-}$flux as observed by the CALET [89], DAMPE [90], and AMS-02 [7] experiments. 
with a Sommerfeld enhanced annihilation cross section of $\sigma_{\bar{\chi} \chi \rightarrow a a} \sim 3.43 \times 10^{-24} \mathrm{~cm}^{3} / \mathrm{sec}$, a very good fit to the DAMPE observations is achieved, wherein the best fit value of the cosmological boost factor $B$ is obtained as 13.1.

\section{CONCLUSION}

The AMS-02 experiment onboard the International Space Station reported an excess of positron fraction beyond the positron energy of $10 \mathrm{GeV}$. While the positron-fraction spectrum below $10 \mathrm{GeV}$ corroborates with the expected spectrum, the excess that peaks around $320 \mathrm{GeV}$ could not be explained by the known astrophysical or other processes, cosmic rays, etc. Therefore, the observed positron-fraction excess could be a signature of new physics or phenomena not fully understood yet.

In this work, we revisit the possibility that dark matter annihilation in the Universe could have caused this excess signature by producing electron-positron pairs through such annihilation processes. To this end, we formulate a new particle DM model based on 2HDM available in the literature and adding to it an extra fermion as a DM candidate and an additional pseudoscalar for the portal. It appears from our calculations that in order to account for the DM annihilation cross section required to satisfy the experimentally observed DM relic density given by the PLANCK collaboration, a pseudoscalar is needed if the extra fermion in the theory is to serve as the DM candidate. It also appears that, for the realization of the observed positron excess within the proposed framework, a leptophilic DM could be a viable candidate. Keeping these in view, we consider the leptophilic $2 \mathrm{HDM}$ that can explain the muon g-2 anomaly while avoiding various experimental and astrophysical constraints as shown in detail. The key feature of the model is the presence of a light bosonic portal through which the required DM relic density, as well as the Sommerfeld enhancement of DM annihilation cross section, can be achieved. It will be an interesting task to search for such a light boson in various low energy experiments.

We have computed the Sommerfeld enhancement in the present framework and made a $\chi^{2}$ fit of the computed positron fraction with the AMS-02 observational results where a boost factor is treated as a free parameter. We obtain a good fit for the theoretical estimation of positron fraction with the boost factor of order 10, which is within the widely accepted ballpark in the literature. We find that the present dark matter model not only satisfactorily explains simultaneously the positron spectrum observed by the AMS-02 and the total $e^{+}+e^{-}$flux determined by AMS-02 and CALET experiments, but it also provides a good fit to $e^{+}+e^{-}$flux reported by DAMPE experiment for a different choice of parameters.

\section{ACKNOWLEDGMENTS}

Three of the authors, S. G., A. D. B., and D. M., would like to thank P. Roy and A. Ray for some useful discussions. A. D. B. would also like to thank Saha Institute of Nuclear Physics for the hospitality. The work of A. D. B. is supported in part by the National Science Foundation of China (Grants No. 11422545 and No. 11947235).
[1] N. Aghanim et al., Planck 2018 results. VI. Cosmological parameters, Astron. Astrophys. 641, A6 (2020).

[2] M. Aguilar et al., First Result from the Alpha Magnetic Spectrometer on the International Space Station: Precision Measurement of the Positron Fraction in Primary Cosmic Rays of 0.5-350 GeV, Phys. Rev. Lett. 110, 141102 (2013).

[3] O. Adriani et al., An anomalous positron abundance in cosmic rays with energies $1.5-100 \mathrm{GeV}$, Nature (London) 458, 607 (2009).

[4] O. Adriani, G. C. Barbarino, G. A. Bazilevskaya, R. Bellotti, M. Boezio, E. A. Bogomolov, L. Bonechi, M. Bongi, V. Bonvicini, and S. Borisov, A statistical procedure for the identification of positrons in the PAMELA experiment, Astropart. Phys. 34, 1 (2010).

[5] L. Accardo et al., High Statistics Measurement of the Positron Fraction in Primary Cosmic Rays of 0.5-500 GeV with the Alpha Magnetic Spectrometer on the International Space Station, Phys. Rev. Lett. 113, 121101 (2014).
[6] M. Aguilar et al., Towards Understanding the Origin of Cosmic-Ray Positrons, Phys. Rev. Lett. 122, 041102 (2019).

[7] M. Aguilar et al., Towards Understanding the Origin of Cosmic-Ray Electrons, Phys. Rev. Lett. 122, 101101 (2019).

[8] M. Linares and M. Kachelriess, Cosmic ray positrons from compact binary millisecond pulsars, J. Cosmol. Astropart. Phys. 02 (2021) 030.

[9] C. Evoli, E. Amato, P. Blasi, and R. Aloisio, Galactic factories of cosmic-ray electrons and positrons, Phys. Rev. D 103, 083010 (2021).

[10] A. U. Abeysekara et al., Extended gamma-ray sources around pulsars constrain the origin of the positron flux at Earth, Science 358, 911 (2017).

[11] L. Feng, R.-Z. Yang, H.-N. He, T.-K. Dong, Y.-Z. Fan, and J. Chang, AMS-02 positron excess: New bounds on dark matter models and hint for primary electron spectrum hardening, Phys. Lett. B 728, 250 (2014).

[12] P. S. Bhupal Dev, D. K. Ghosh, N. Okada, and I. Saha, Neutrino mass and Dark Matter in light of recent AMS-02 results, Phys. Rev. D 89, 095001 (2014). 
[13] Y. Bai, J. Berger, and S. Lu, Supersymmetric resonant dark matter: A thermal model for the AMS-02 positron excess, Phys. Rev. D 97, 115012 (2018).

[14] A. Das, B. Dasgupta, and A. Ray, Galactic positron excess from selectively enhanced Dark Matter annihilation, Phys. Rev. D 101, 063014 (2020).

[15] I. Cholis and D. Hooper, Dark Matter and pulsar origins of the rising cosmic ray positron fraction in light of new data from AMS, Phys. Rev. D 88, 023013 (2013).

[16] A. Ibarra, A. S. Lamperstorfer, and J. Silk, Dark Matter annihilations and decays after the AMS-02 positron measurements, Phys. Rev. D 89, 063539 (2014).

[17] H.-B. Jin, Y.-L. Wu, and Y.-F. Zhou, Implications of the first AMS-02 measurement for dark matter annihilation and decay, J. Cosmol. Astropart. Phys. 11 (2013) 026.

[18] S. Y. Ayazi, S. M. Firouzabadi, and S. P. Zakeri, Freeze-in production of Fermionic Dark Matter with Pseudo-scalar and Phenomenological Aspects, J. Phys. G 43, 095006 (2016).

[19] S.-J. Lin, Q. Yuan, and X.-J. Bi, Quantitative study of the AMS-02 electron/positron spectra: Implications for pulsars and dark matter properties, Phys. Rev. D 91, 063508 (2015).

[20] Y.-C. Ding, Y.-L. Ku, C.-C. Wei, and Y.-F. Zhou, Consistent explanation for the cosmic-ray positron excess in $\mathrm{p}$-wave Sommerfeld-enhanced dark matter annihilation, J. Cosmol. Astropart. Phys. 09 (2021) 005.

[21] F. S. Queiroz and C. Siqueira, Explaining the AMS positron excess via Right-handed Neutrinos, Phys. Rev. D 101, 075007 (2020).

[22] S. Profumo, F. Queiroz, and C. Siqueira, Has AMS-02 observed two-component dark matter?, J. Phys. G 48, 015006 (2021).

[23] K. Belotsky, M. Khlopov, C. Kouvaris, and M. Laletin, High energy positrons and gamma radiation from decaying constituents of a two-component dark atom model, Int. J. Mod. Phys. D 24, 1545004 (2015).

[24] K. Belotsky et al., Decaying dark atom constituents and cosmic positron excess, Adv. High Energy Phys. 2014, 214258 (2014).

[25] Y. Farzan and M. Rajaee, Dark matter decaying into millicharged particles as a solution to AMS 02 positron excess, J. Cosmol. Astropart. Phys. 04 (2019) 040.

[26] Y. G. Kim, K. Y. Lee, and S. Shin, Singlet fermionic dark matter, J. High Energy Phys. 05 (2008) 100.

[27] S. Hoof, A. Geringer-Sameth, and R. Trotta, A global analysis of dark matter signals from 27 dwarf spheroidal galaxies using 11 years of Fermi-LAT observations, J. Cosmol. Astropart. Phys. 02 (2020) 012.

[28] N. Arkani-Hamed, D. P. Finkbeiner, T. R. Slatyer, and N. Weiner, A theory of dark matter, Phys. Rev. D 79, 015014 (2009).

[29] E. J. Chun and J.-C. Park, Dark matter and sub-GeV hidden U(1) in GMSB models, J. Cosmol. Astropart. Phys. 02 (2009) 026.

[30] A. Broggio, E. J. Chun, M. Passera, K. M. Patel, and S. K. Vempati, Limiting two-Higgs-doublet models, J. High Energy Phys. 11 (2014) 058.

[31] H. Abdallah et al., Search for Dark Matter Annihilations Towards the Inner Galactic Halo from 10 Years of Observations with H.E.S.S, Phys. Rev. Lett. 117, 111301 (2016).
[32] S. Profumo, F. S. Queiroz, J. Silk, and C. Siqueira, Searching for secluded dark matter with H.E.S.S., Fermi-LAT, and planck, J. Cosmol. Astropart. Phys. 03 (2018) 010.

[33] M. Cirelli, G. Corcella, A. Hektor, G. Hütsi, M. Kadastik, P. Panci, M. Raidal, F. Sala, and A. Strumia, PPPC 4 DM ID: A poor particle physicist cookbook for dark matter indirect detection, J. Cosmol. Astropart. Phys. 03 (2011) 051; Erratum, J. Cosmol. Astropart. Phys. 10 (2012) E01.

[34] D. Goncalves, P. A. N. Machado, and J. M. No, Simplified models for dark matter face their consistent completions, Phys. Rev. D 95, 055027 (2017).

[35] K.-C. Yang, Fermionic dark matter through a light pseudoscalar portal: Hints from the DAMA results, Phys. Rev. D 94, 035028 (2016).

[36] G. Arcadi, M. Lindner, F. S. Queiroz, W. Rodejohann, and S. Vogl, Pseudoscalar mediators: A WIMP model at the neutrino floor, J. Cosmol. Astropart. Phys. 03 (2018) 042.

[37] M. J. Dolan, F. Kahlhoefer, C. McCabe, and K. SchmidtHoberg, A taste of dark matter: Flavour constraints on pseudoscalar mediators, J. High Energy Phys. 03 (2015) 171; Erratum, J. High Energy Phys. 07 (2015) 103.

[38] M. Bauer, U. Haisch, and F. Kahlhoefer, Simplified dark matter models with two Higgs doublets: I. Pseudoscalar mediators, J. High Energy Phys. 05 (2017) 138.

[39] M. Bauer, M. Klassen, and V. Tenorth, Universal properties of pseudoscalar mediators in dark matter extensions of 2HDMs, J. High Energy Phys. 07 (2018) 107.

[40] S. Ipek, D. McKeen, and A. E. Nelson, A renormalizable model for the galactic center gamma ray excess from dark matter annihilation, Phys. Rev. D 90, 055021 (2014).

[41] J. M. No, Looking through the pseudoscalar portal into dark matter: Novel mono-Higgs and mono-Z signatures at the LHC, Phys. Rev. D 93, 031701 (2016).

[42] G. Arcadi, G. Busoni, T. Hugle, and V. T. Tenorth, Comparing 2HDM + scalar and pseudoscalar simplified models at LHC, J. High Energy Phys. 06 (2020) 098.

[43] G. C. Branco, P. M. Ferreira, L. Lavoura, M. N. Rebelo, M. Sher, and J. P. Silva, Theory and phenomenology of twoHiggs-doublet models, Phys. Rep. 516, 1 (2012).

[44] D. Eriksson, J. Rathsman, and O. Stal, 2HDMC: Two-Higgs-doublet model calculator physics and manual, Comput. Phys. Commun. 181, 189 (2010).

[45] E. J. Chun, S. Dwivedi, T. Mondal, B. Mukhopadhyaya, and S. K. Rai, Reconstructing heavy Higgs boson masses in a type $\mathrm{X}$ two-Higgs-doublet model with a light pseudoscalar particle, Phys. Rev. D 98, 075008 (2018).

[46] E. J. Chun, S. Dwivedi, T. Mondal, and B. Mukhopadhyaya, Reconstructing a light pseudoscalar in the Type-X Two Higgs doublet model, Phys. Lett. B 774, 20 (2017).

[47] T. Abe, M. Fujiwara, and J. Hisano, Loop corrections to dark matter direct detection in a pseudoscalar mediator dark matter model, J. High Energy Phys. 02 (2019) 028.

[48] A. M. Sirunyan et al., Search for an exotic decay of the Higgs boson to a pair of light pseudoscalars in the final state of two muons and two $\tau$ leptons in proton-proton collisions at $\sqrt{s}=13 \mathrm{TeV}$, J. High Energy Phys. 11 (2018) 018.

[49] A. M. Sirunyan et al., A search for pair production of new light bosons decaying into muons in proton-proton collisions at 13 TeV, Phys. Lett. B 796, 131 (2019). 
[50] J. Cao, P. Wan, L. Wu, and J. M. Yang, Lepton-specific twoHiggs doublet model: Experimental constraints and implication on Higgs phenomenology, Phys. Rev. D 80, 071701 (2009).

[51] L. Wang and X.-F. Han, A light pseudoscalar of 2HDM confronted with muon g-2 and experimental constraints, J. High Energy Phys. 05 (2015) 039.

[52] T. Abe, R. Sato, and K. Yagyu, Lepton-specific two Higgs doublet model as a solution of muon $\mathrm{g}-2$ anomaly, J. High Energy Phys. 07 (2015) 064.

[53] E. J. Chun and J. Kim, Leptonic precision test of leptophilic two-Higgs-doublet model, J. High Energy Phys. 07 (2016) 110.

[54] B. Abi et al., Measurement of the Positive Muon Anomalous Magnetic Moment to 0.46 ppm, Phys. Rev. Lett. 126, 141801 (2021).

[55] R. Aaij et al., Measurement of the $B_{s}^{0} \rightarrow \mu^{+} \mu^{-}$Branching Fraction and Effective Lifetime and Search for $B^{0} \rightarrow \mu^{+} \mu^{-}$ Decays, Phys. Rev. Lett. 118, 191801 (2017).

[56] J. Beacham et al., Physics beyond colliders at CERN: Beyond the standard model working group report, J. Phys. G 47, 010501 (2020).

[57] B. Batell, N. Lange, D. McKeen, M. Pospelov, and A. Ritz, Muon anomalous magnetic moment through the leptonic Higgs portal, Phys. Rev. D 95, 075003 (2017).

[58] L. Marsicano, M. Battaglieri, A. Celentano, R. De Vita, and Y.-M. Zhong, Probing leptophilic dark sectors at electron beam-dump facilities, Phys. Rev. D 98, 115022 (2018).

[59] M. Davier and H. N. Ngoc, An unambiguous search for a light Higgs boson, Phys. Lett. B 229, 150 (1989).

[60] J. D. Bjorken, S. Ecklund, W. R. Nelson, A. Abashian, C. Church, B. Lu, L. W. Mo, T. A. Nunamaker, and P. Rassmann, Search for neutral metastable penetrating particles produced in the SLAC beam dump, Phys. Rev. D 38, 3375 (1988).

[61] J. P. Lees et al., Search for a Dark Leptophilic Scalar in $e^{+} e^{-}$Collisions, Phys. Rev. Lett. 125, 181801 (2020).

[62] C.-Y. Chen, M. Pospelov, and Y.-M. Zhong, Muon beam experiments to probe the dark sector, Phys. Rev. D 95, 115005 (2017).

[63] A. Berlin, S. Gori, P. Schuster, and N. Toro, Dark sectors at the fermilab seaquest experiment, Phys. Rev. D 98, 035011 (2018).

[64] E. J. Chun and T. Mondal, Searching for a light Higgs boson via the yukawa process at lepton colliders, Phys. Lett. B 802, 135190 (2020).

[65] E. J. Chun and T. Mondal, Leptophilic bosons and muon g-2 at lepton colliders, J. High Energy Phys. 07 (2021) 044.

[66] R. Aaij et al., Search for long-lived scalar particles in $B^{+} \rightarrow K^{+} \chi\left(\mu^{+} \mu^{-}\right)$decays, Phys. Rev. D 95, 071101 (2017).

[67] A. Filimonova, R. Schäfer, and S. Westhoff, Probing dark sectors with long-lived particles at BELLE II, Phys. Rev. D 101, 095006 (2020).

[68] S. Alekhin et al., A facility to search for hidden particles at the CERN SPS: The SHiP physics case, Rep. Prog. Phys. 79, 124201 (2016).
[69] D. Curtin et al., Long-lived particles at the energy frontier: The MATHUSLA physics case, Rep. Prog. Phys. 82, 116201 (2019).

[70] N. F. Bell, Y. Cai, J. B. Dent, R. K. Leane, and T. J. Weiler, Enhancing darkmatter annihilation rates with dark bremsstrahlung, Phys. Rev. D 96, 023011 (2017).

[71] S. Dutta, A. Goyal, and M. P. Singh, Lepto-philic 2-HDM + singlet scalar portal induced fermionic dark matter, J. High Energy Phys. 07 (2019) 076.

[72] E. W. Kolb and M. S. Turner, The Early Universe, Front. Phys. 69, 1 (1990).

[73] A. Sommerfeld, Über die beugung und bremsung der elektronen, Ann. Phys. (Berlin) 403, 257 (1931).

[74] J. L. Feng, M. Kaplinghat, and H.-B. Yu, Sommerfeld enhancements for thermal relic Dark Matter, Phys. Rev. D 82, 083525 (2010).

[75] P. Agrawal, A. Parikh, and M. Reece, Systematizing the effective theory of self-interacting dark matter, J. High Energy Phys. 10 (2020) 191.

[76] S. Cassel, Sommerfeld factor for arbitrary partial wave processes, J. Phys. G 37, 105009 (2010).

[77] T. R. Slatyer, The sommerfeld enhancement for dark matter with an excited state, J. Cosmol. Astropart. Phys. 02 (2010) 028.

[78] M. Cirelli, R. Franceschini, and A. Strumia, Minimal dark matter predictions for galactic positrons, anti-protons, photons, Nucl. Phys. B800, 204 (2008).

[79] E. A. Baltz and J. Edsjo, Positron propagation and fluxes from neutralino annihilation in the halo, Phys. Rev. D 59, 023511 (1998).

[80] T. Delahaye, R. Lineros, F. Donato, N. Fornengo, J. Lavalle, P. Salati, and R. Taillet, Galactic secondary positron flux at the Earth, Astron. Astrophys. 501, 821 (2009).

[81] M. Boudaud, M. Cirelli, G. Giesen, and P. Salati, A fussy revisitation of antiprotons as a tool for Dark Matter searches, J. Cosmol. Astropart. Phys. 05 (2015) 013.

[82] J. Lavalle, Q. Yuan, D. Maurin, and X.-J. Bi, Full calculation of clumpiness boost factors for antimatter cosmic rays in the light of Lambda-CDM N-body simulation results. Abandoning hope in clumpiness enhancement?, Astron. Astrophys. 479, 427 (2008).

[83] P. Brun, G. Bertone, J. Lavalle, P. Salati, and R. Taillet, Antiproton and positron signal enhancement in dark matter mini-spikes scenarios, Phys. Rev. D 76, 083506 (2007).

[84] V. Berezinsky, V. Dokuchaev, and Y. Eroshenko, Smallscale clumps in the galactic halo and dark matter annihilation, Phys. Rev. D 68, 103003 (2003).

[85] J. Lavalle, J. Pochon, P. Salati, and R. Taillet, Clumpiness of dark matter and positron annihilation signal: Computing the odds of the galactic lottery, Astron. Astrophys. 462, 827 (2007).

[86] H.-S. Goh, L. J. Hall, and P. Kumar, The leptonic Higgs as a messenger of dark matter, J. High Energy Phys. 05 (2009) 097.

[87] M. Ackermann et al., Measurement of Separate CosmicRay Electron and Positron Spectra with the Fermi Large Area Telescope, Phys. Rev. Lett. 108, 011103 (2012). 
[88] O. Adriani et al., Cosmic-Ray Positron Energy Spectrum Measured by PAMELA, Phys. Rev. Lett. 111, 081102 (2013).

[89] O. Adriani et al., Extended Measurement of the CosmicRay Electron and Positron Spectrum from $11 \mathrm{GeV}$ to 4.8 TeV with the Calorimetric Electron Telescope on the
International Space Station, Phys. Rev. Lett. 120, 261102 (2018).

[90] G. Ambrosi et al., Direct detection of a break in the teraelectronvolt cosmic-ray spectrum of electrons and positrons, Nature (London) 552, 63 (2017). 